\title{
Clinical Study \\ HDL-C Response Variability to Niacin ER in US Adults
}

\author{
Jennifer B. Christian, ${ }^{1}$ Eric J. Olson, ${ }^{2}$ Jeffery K. Allen, ${ }^{3}$ and Kimberly A. Lowe ${ }^{4}$ \\ ${ }^{1}$ GlaxoSmithKline, Clinical Effectiveness \& Safety, 5 Moore Drive, B.3116, Durham, NC 27709-3398, USA \\ ${ }^{2}$ GlaxoSmithKline, Heart Failure DPU, Medicines Discovery and Development, King of Prussia, PA 19406, USA \\ ${ }^{3}$ GlaxoSmithKline, Worldwide Epidemiology, Durham, NC 27709, USA \\ ${ }^{4}$ Exponent Health Sciences, Bellevue, WA 98004, USA
}

Correspondence should be addressed to Jennifer B. Christian; jennifer.b.christian@gsk.com

Received 20 December 2012; Accepted 28 January 2013

Academic Editor: Francisco Blanco-Vaca

Copyright (C) 2013 Jennifer B. Christian et al. This is an open access article distributed under the Creative Commons Attribution License, which permits unrestricted use, distribution, and reproduction in any medium, provided the original work is properly cited.

\begin{abstract}
Background. Niacin is the most effective treatment currently available for raising HDL-C levels. Objective. To evaluate if gender and baseline lipid levels have an effect on the HDL-C response of niacin ER and to identify factors that predict response to niacin ER at the $500 \mathrm{mg}$ dose. Material and Methods. The change in HDL-C effect between baseline and follow-up levels was quantified in absolute change as well as dichotomized into high versus low response (high response was defined as an HDL-C effect of $>15 \%$ increase and low response was HDL-C $<5 \%$ ) in a sample of 834 individuals. Results. Both males and females with low HDL-C levels at baseline exhibited a response to treatment in the multivariate model (males, HDL-C <40 mg/dL: OR = 5.18, 95\% CI: 2.36-11.39; females, HDL-C $<50 \mathrm{mg} / \mathrm{dL}$ : OR $=5.40,95 \% \mathrm{CI}: 1.84-15.79)$. There was also a significant difference in the mean HDL-C effect between baseline and follow-up HDL-C levels in the $500 \mathrm{mg}$ niacin ER dose group for both males (mean HDL-C effect $=0.08$, $P<0.001$ ) and females (mean HDL-C effect $=0.10, P=0.019$ ). Conclusion. Baseline HDL-C levels are the biggest predictor of response to niacin ER treatment for both males and females among the factors evaluated.
\end{abstract}

\section{Introduction}

High-density lipoprotein cholesterol (HDL-C), also referred to as the "good cholesterol," is a known antioxidant, antithrombotic, and antiinflammatory, with properties that promote the removal of cellular cholesterol [1]. Individuals with low HDL-C levels may be at an increased risk of coronary heart disease (CHD) [2], and it has been shown in observational studies that the risk of CHD is reduced by $2 \%$ $3 \%$ for every $1 \mathrm{mg} / \mathrm{dL}$ increase in HDL-C [3]. Approximately $16 \%$ of adults in the United States have low HDL-C levels (25\% of males and $7 \%$ of females) [4], while $11 \%$ of males and $4 \%$ of females of 19 years of age or younger have low HDL-C [5].

Low HDL-C is managed by three classes of dyslipidemic pharmacotherapy: niacin, statins, and fibric acid derivatives. Of these, niacin has been shown to increase HDL-C levels by as much as $35 \%$ in higher doses, which makes it the most effective pharmaceutical treatment currently available for raising HDL-C [6-9]. In addition, extended-release (ER) high-dose niacin has been shown to significantly reduce carotid atherosclerosis within 12 months among statintreated individuals with low HDL-C who had either type II diabetes with coronary heart disease or carotid/peripheral atherosclerosis [9]. However, recently published results from the Atherothrombosis Intervention in Metabolic Syndrome with Low HDL Cholesterol/High Triglyceride and Impact on Global Health Outcomes (AIM-HIGH) study showed that high-dose niacin ER did not provide additional reduction in risk of cardiovascular-related events beyond what is observed with statin therapy alone [4]. In this modest sized trial, 3,414 subjects were randomized to niacin ER or placebo while their low-density lipoprotein cholesterol (LDL-C) levels were maintained to a median of $<70 \mathrm{mg} / \mathrm{dL}$. Importantly, the lipid community still awaits the results of a second larger niacin ER study (HSP2 THRIVE) to understand where the potential value of niacin therapy may reside in $\mathrm{CV}$ risk reduction, if it does at all. Therefore, due to the findings of the AIM-HIGH study and until the results of the HSP2 THRIVE results are revealed (in addition to the poor tolerability of niacin itself), 
administration of niacin to those who stand to achieve the most lipid benefit is important.

Therefore, our objectives were to evaluate the extent to which gender and baseline lipid levels may have an effect on the HDL-C response of niacin ER and to identify factors that predict greater response to niacin ER.

\section{Materials and Methods}

2.1. Data Source and Description of the Study Population. This is a retrospective cohort study using medical claims that occurred between January 1, 2000, and March 31, 2008, from the Optum Impact National Managed Care Benchmark Database (OptumInsight, Eden Prairie, MN, USA). This database includes de-identified claims for inpatient and outpatient services, laboratory results, and pharmacy fills for privately insured patients, including several hundred thousand Managed Medicare and Medicare Advantage members. It is a commonly used and reliable data source for epidemiologic research in the United States [7, 9-12]. Database administrators perform multiple cross-checks to ensure data accuracy. Such practices include tracking of historical trends to identify anomalies and outliers. Database metrics are run after each update to cross-check the data and ensure it meets known population distributions. Source-to-target validation of data quantities is also completed and prescribed rules for researching and discarding nonconforming data are followed. The patients included in the database are representative of privately insured patients in all 50 states. A large proportion of the lab results come from two major lab vendors.

The sample for the current study includes patients $\geq 18$ years of age who met the following criteria: (1) concurrent medical and prescription coverage for the study period for at least two months while taking niacin ER, (2) a prescription of at least 28 days of niacin ER filled during the study period, (3) continuous eligibility for six months prior to the first niacin ER prescription and $\geq 29$ days after the first prescription at the desired dose of niacin ER, (4) HDL-C measurements were available $\leq 6$ months prior to and $\geq 29$ days after filling the first prescription of niacin ER, and (5) gender was provided in the claims record.

2.2. Niacin ER Dose Levels. Prescription niacin ER was measured at $500 \mathrm{mg}$ and $1000 \mathrm{mg}$ doses. The two niacin ER dose groups were mutually exclusive, and all study participants were taking the niacin ER in combination with a statin. Individuals who qualified for the study in more than one dose group were categorized into the highest dose group for the purposes of this study.

2.3. Baseline Lipid Levels. Baseline lipid levels were taken within a six-month period prior to starting niacin ER at any dose and were dichotomized into the following groups for analysis: $\mathrm{HDL}-\mathrm{C}$ levels for males and females combined ( $<40 \mathrm{mg} / \mathrm{dL}$ versus $\geq 40 \mathrm{mg} / \mathrm{dL}$ ), HDL-C levels for males only (HDL-C $<40 \mathrm{mg} / \mathrm{dL}$ versus $\geq 40 \mathrm{mg} / \mathrm{dL}$ ), HDL-C levels for females only (HDL-C $<50 \mathrm{mg} / \mathrm{dL}$ versus $\geq 50 \mathrm{mg} / \mathrm{dL}$ ), LDLC levels $(<130 \mathrm{mg} / \mathrm{dL}$ versus $\geq 130 \mathrm{mg} / \mathrm{dL})$, triglyceride $(\mathrm{TG})$ levels $<150 \mathrm{mg} / \mathrm{dL}$ versus $\geq 150 \mathrm{mg} / \mathrm{dL}$ ), and total cholesterol (TC) levels ( $<200 \mathrm{mg} / \mathrm{dL}$ versus $\geq 200 \mathrm{mg} / \mathrm{dL})$.

2.4. Change in HDL Effect. Follow-up HDL-C levels were included in the analysis after the first month of treatment with niacin ER (at the specific dose of interest) through 5 days after termination of niacin ER. The distribution of change in HDL-C effect was evaluated with $5 \%$ intervals by gender and also by dose of niacin ER. For the purposes of statistical analysis, the change in HDL-C effect was measured in two ways: (1) as a continuous variable and (2) dichotomized into high versus low response, in which high response was defined as an HDL-C effect of $>15 \%$ and low response was HDL-C $<5 \%$. These cut points were selected based on a combination of the distribution of HDL-C response in our sample and a meaningful clinical response to niacin ER.

2.5. Statistical Analysis. Baseline demographic characteristics and lipid levels were summarized as frequencies and percentages. The change in HDL-C effect between baseline and followup was quantified by the difference and divided by the baseline value and then summarized using mean values and standard deviations. The Student's $t$-test was used to test the null hypothesis that there was no difference in the mean HDL-C effect by dichotomous categorical variables. The $F$ statistic was used to test the null hypothesis that there was no difference in the mean HDL-C effect across variables with three or more categories. Crude and adjusted multivariate logistic regression models were then developed to evaluate $\mathrm{HDL}-\mathrm{C}$ response to niacin ER at the $500 \mathrm{mg}$ dose level by calculating an odds ratio (OR) and 95\% confidence interval (CI). As described above, our main analysis defined high responders as individuals with an HDL-C effect of $>15 \%$ and low responders were defined as individuals with a $<5 \% \mathrm{HDL}-$ $\mathrm{C}$ effect. The outcomes in these models were high response (yes or no).

A sensitivity analysis was also conducted where high response was defined as a combination of HDL-C rise of $>15 \%$ and LDL-C lowering effect of $>-15 \%$, and low response was defined as $<5 \%$ HDL-C effect and LDL-C effect was $>-15 \%$. This restricted definition was used to ensure that subjects were taking their combination medications of niacin and a statin but not achieving the anticipated rise in HDL-C. Crude and adjusted multivariate logistic regression models were developed using this restricted definition to evaluate HDL-C response to niacin ER at the $500 \mathrm{mg}$ dose level.

\section{Results}

Our sample included a total of 834 study participants (680 at the $500 \mathrm{mg}$ niacin ER dose level, and 154 at the $1000 \mathrm{mg}$ niacin ER dose level). Table 1 summarizes the baseline characteristics and lipid levels of the study participants by niacin ER dose level. The majority of study participants in each niacin ER dose level were 41 to 65 years of age, male, had HDL-C levels $<40 \mathrm{mg} / \mathrm{dL}$, had baseline LDL-C levels $<130 \mathrm{mg} / \mathrm{dL}$, and had baseline triglyceride levels $\geq 150 \mathrm{mg} / \mathrm{dL}$. The majority of study participants in the $500 \mathrm{mg}$ niacin ER dose group had baseline 
total cholesterol levels $\geq 200 \mathrm{mg}$, while the majority of the study participants in the $1000 \mathrm{mg}$ niacin ER dose group had baseline total cholesterol levels $<200 \mathrm{mg} / \mathrm{dL}$. The proportion of study participants who had diabetes, stroke (within six months), hypertension (within six months), and ischemic heart disease (within six months) were similar in both dose groups. The average persistence of niacin ER was 193.6 days in the $500 \mathrm{mg}$ dose group and $192.1 \mathrm{in}$ the $1000 \mathrm{mg}$ dose group.

Figure 1 illustrates the distribution of HDL-C effect by niacin ER dose and baseline lipid levels, and Table 2 reports the results of the inferential statistics of the overall HDL-C effect by niacin ER dose. There was a statistically significant change in the mean HDL-C effect between baseline and follow-up HDL-C levels in the $500 \mathrm{mg}$ niacin ER dose group for both males (mean HDL-C effect $=0.08, P<0.001$ ) and females (mean HDL-C effect $=0.10, P=0.019$ ). Similar results were observed in the $1000 \mathrm{mg}$ niacin ER dose group for males (mean HDL-C effect $=0.08, P=0.029$ ) and females (mean HDL-C effect $=0.19, P=0.048$ ). Females had statistically significantly higher levels of both baseline and follow-up HDL-C levels than males in both niacin ER dose groups. In addition, there was a statistically significant difference in the HDL-C effect observed among females across the niacin ER dose groups $(P=0.021)$. This trend was not observed among males $(P=0.768)$. There was a statistically significant change in the mean HDL-C effect between baseline and follow-up HDL-C levels in both niacin ER dose groups among individuals of 41 to 65 years of age (mean HDL-C effect in the $500 \mathrm{mg}$ dose group $=0.08, P<$ 0.001 ; mean HDL-C effect in the $1000 \mathrm{mg}$ dose group $=0.12$, $P=0.008$ ), but not among individuals in the 18 to 40 or $\geq 66$ age groups. The mean HDL-C levels were statistically significantly higher in the $>66$-year age group than the other age groups at baseline $(P=0.03)$ and followup $(P=0.018)$ among participants in the $500 \mathrm{mg}$ niacin ER dose group but not in the $1000 \mathrm{mg}$ niacin ER dose group. The change in the mean HDL-C effect between baseline and follow-up HDL-C levels was statistically significant for individuals with HDL-C levels $<40 \mathrm{mg} / \mathrm{dL}$ in the $500 \mathrm{mg}$ niacin ER dose group (mean HDL-C effect $=0.12, P<0.001)$ and also for individuals in the $1000 \mathrm{mg}$ niacin ER dose group (mean HDL-C effect = $0.13, P<0.001)$. There was no change in the HDL-C effect among individuals with HDL-C levels $\geq 40 \mathrm{mg} / \mathrm{dL}$ in either niacin ER dose group. When baseline LDL-C levels were stratified at $130 \mathrm{mg} / \mathrm{dL}$, there was a statistically significant difference in the mean HDL-C effect between baseline and follow-up HDL-C levels among individuals who had LDL-C levels $<130 \mathrm{mg} / \mathrm{dL}$ (mean HDL-C effect $=0.07, P=0.025$ ) and among those who had LDL-C $\geq 130$ (mean HDL-C effect $=0.08, P=0.031$ ) in the $500 \mathrm{mg}$ niacin ER dose. Among individuals in the $1000 \mathrm{mg}$ niacin ER dose group, only individuals with LDL-C levels $<130$ showed a statistically significant improvement in the mean HDL-C effect (mean HDL-C effect $=0.12, P=0.008$ ). The mean HDL-C effect was statistically significantly higher among individuals with triglyceride levels $\geq 150 \mathrm{mg} / \mathrm{dL}$ (mean HDL-C effect $=0.08$, $P<0.001)$ in the $500 \mathrm{mg}$ niacin ER dose group and among individuals in that TG category in the $1000 \mathrm{mg}$ niacin ER dose group (HDL-C effect $=0.12, P=0.005$ ). There were
TABLE 1: Baseline characteristics and lipid levels ${ }^{1}$.

\begin{tabular}{|c|c|c|}
\hline Demographics and lipids & $\begin{array}{c}500 \text { mg niacin } \\
\text { ER dose } \\
(n=680) \\
n(\%)\end{array}$ & $\begin{array}{c}1000 \text { mg niacin } \\
\text { ER dose } \\
(n=154) \\
n(\%)\end{array}$ \\
\hline \multicolumn{3}{|l|}{ Age (years) } \\
\hline 18 to 40 & $79(11.6)$ & $12(7.8)$ \\
\hline 41 to 65 & $516(75.9)$ & $131(85.1)$ \\
\hline$\geq 66$ & $85(12.5)$ & $11(7.1)$ \\
\hline \multicolumn{3}{|l|}{ Gender } \\
\hline Male & $480(70.6)$ & $120(77.9)$ \\
\hline Female & $200(29.4)$ & $34(22.1)$ \\
\hline \multicolumn{3}{|l|}{ HDL-C mg/dL (both genders) } \\
\hline$<40$ & $419(61.6)$ & $87(56.5)$ \\
\hline 40 to 59 & $213(31.3)$ & $59(38.3)$ \\
\hline$\geq 60$ & $44(6.5)$ & $8(5.2)$ \\
\hline \multicolumn{3}{|l|}{ Baseline LDL-C mg/dL } \\
\hline$<130$ & $266(39.1)$ & $87(56.5)$ \\
\hline$\geq 130$ & $178(26.2)$ & $34(22.1)$ \\
\hline Unknown & $236(34.7)$ & $33(21.4)$ \\
\hline \multicolumn{3}{|l|}{ Baseline TG mg/dL } \\
\hline$<150$ & $192(28.2)$ & $60(39.0)$ \\
\hline$\geq 150$ & $483(71.0)$ & $93(60.4)$ \\
\hline Unknown & $5(0.7)$ & $1(0.7)$ \\
\hline \multicolumn{3}{|l|}{ Baseline TC mg/dL } \\
\hline$<200$ & $301(44.3)$ & $96(62.3)$ \\
\hline$\geq 200$ & $375(55.2)$ & $57(37.0)$ \\
\hline Unknown & $4(0.6)$ & $1(0.7)$ \\
\hline \multicolumn{3}{|l|}{ Comorbidities } \\
\hline Diabetes & $188(27.7)$ & $41(26.6)$ \\
\hline Stroke (within 6 months) & $26(3.8)$ & $10(6.5)$ \\
\hline $\begin{array}{l}\text { Hypertension } \\
\text { (within } 6 \text { months) }\end{array}$ & $46(6.8)$ & $7(4.6)$ \\
\hline $\begin{array}{l}\text { Ischemic heart disease } \\
\text { (within } 6 \text { months) }\end{array}$ & $130(19.1)$ & $32(20.8)$ \\
\hline \multicolumn{3}{|l|}{ Medication use } \\
\hline Prestatin users & $258(37.9)$ & $64(41.6)$ \\
\hline Prefibric acid users & $82(12.1)$ & $12(7.8)$ \\
\hline \multicolumn{3}{|l|}{ Persistence } \\
\hline Average persistence (days) & 193.6 & 192.1 \\
\hline$\geq 365$ days persistence & 79 (11.6) & $17(11.0)$ \\
\hline$<365$ days persistence & $601(88.4)$ & $137(89.0)$ \\
\hline
\end{tabular}

${ }^{1}$ Lipid levels were measured within six months prior to any niacin ER use. Definition of lipids: HDL-C: high-density lipoprotein cholesterol; LDL-C: low-density lipoprotein cholesterol; TG: triglycerides; TC: total cholesterol.

no significant changes in the HDL-C effect in either niacin ER dose group among individuals with triglyceride levels $<150 \mathrm{mg} / \mathrm{dL}$. There was a statistically significant difference in the mean HDL-C effect among individuals with total cholesterol levels $<200 \mathrm{mg} / \mathrm{dL}$ (mean HDL-C effect $=0.09$, 
TABLE 2: Overall HDL effect by niacin ER dose.

\begin{tabular}{|c|c|c|c|c|c|c|c|c|c|c|c|}
\hline \multirow[b]{2}{*}{$\begin{array}{l}\text { Demographics } \\
\text { and lipids }\end{array}$} & \multirow[b]{2}{*}{$n$} & \multicolumn{3}{|c|}{$500 \mathrm{mg}$ niacin ER dose } & \multicolumn{7}{|c|}{1000 mg niacin ER dose } \\
\hline & & $\begin{array}{c}\text { Baseline } \\
\text { HDL-C } \\
\text { levels } \\
\text { Mean (SD) }\end{array}$ & $\begin{array}{l}\text { Follow-up } \\
\text { HDL-C } \\
\text { levels } \\
\text { Mean (SD) }\end{array}$ & $\begin{array}{c}\text { HDL-C } \\
\text { effect } \\
\text { Mean (SD) }\end{array}$ & $P$ value $^{\mathrm{a}}$ & $n$ & $\begin{array}{c}\text { Baseline } \\
\text { HDL-C } \\
\text { levels } \\
\text { Mean (SD) }\end{array}$ & $\begin{array}{l}\text { Follow-up } \\
\text { HDL-C } \\
\text { levels } \\
\text { Mean (SD) }\end{array}$ & $\begin{array}{c}\text { HDL-C } \\
\text { effect } \\
\text { Mean (SD) }\end{array}$ & $P$ value $^{\mathrm{a}}$ & $P$ value $^{\mathrm{b}}$ \\
\hline \multicolumn{12}{|l|}{ Gender } \\
\hline Males & 480 & $37.1(0.4)$ & $39.4(0.4)$ & $0.08(0.01)$ & $<0.001$ & 120 & $39.0(0.9)$ & $41.6(0.9)$ & $0.08(0.02)$ & 0.029 & $0.768^{\mathrm{b}}$ \\
\hline Females & 200 & $45.5(1.1)$ & $49.3(1.2)$ & $0.10(0.01)$ & 0.019 & 34 & $48.5(2.6)$ & $57.4(3.6)$ & $0.19(0.03)$ & 0.048 & $0.021^{\mathrm{b}}$ \\
\hline$P$ value ${ }^{c}$ & & $<0.001$ & $<0.001$ & 0.221 & & & 0.001 & $<0.001$ & 0.009 & & $0.053^{\mathrm{d}}$ \\
\hline \multicolumn{12}{|l|}{ Age (years) } \\
\hline 18 to 40 & 79 & $37.9(1.2)$ & $40.0(1.1)$ & $0.10(0.04)$ & 0.196 & 12 & $38.9(2.9)$ & $40.4(1.7)$ & $0.07(0.05)$ & 0.659 & $0.660^{\mathrm{b}}$ \\
\hline 41 to 65 & 516 & $39.4(0.5)$ & $42.2(0.6)$ & $0.08(0.01)$ & $<0.001$ & 131 & $41.3(1.1)$ & $45.8(1.3)$ & $0.12(0.02)$ & 0.008 & $0.057^{\mathrm{b}}$ \\
\hline$\geq 66$ & 85 & $42.5(1.4)$ & $45.5(1.4)$ & $0.08(0.02)$ & 0.126 & 11 & $41.2(2.2)$ & $41.9(1.8)$ & $0.03(0.05)$ & 0.803 & $0.335^{\mathrm{b}}$ \\
\hline$P$ value $^{\mathrm{d}}$ & & 0.032 & 0.018 & 0.842 & & & 0.799 & 0.343 & 0.242 & & $0.613^{\mathrm{d}}$ \\
\hline \multicolumn{12}{|l|}{$\begin{array}{l}\text { HDL-C mg/dL } \\
\text { (both genders) }\end{array}$} \\
\hline 40 & 423 & $32.8(0.3)$ & $36.3(0.4)$ & $0.12(0.01)$ & $<0.001$ & 87 & $33.5(0.5)$ & $37.7(0.8)$ & $0.13(0.02)$ & $<0.001$ & $0.437^{\mathrm{b}}$ \\
\hline$\geq 40$ & 257 & $50.8(0.7)$ & $52.3(0.8)$ & $0.03(0.01)$ & 0.168 & 67 & $50.9(1.2)$ & $54.7(1.9)$ & $0.07(0.02)$ & 0.096 & $0.094^{\mathrm{b}}$ \\
\hline$P$ value ${ }^{c}$ & & $<0.001$ & $<0.001$ & $<0.001$ & & & $<0.001$ & $<0.001$ & 0.037 & & $0^{\mathrm{d}}$ \\
\hline \multicolumn{12}{|l|}{ LDL-C mg/dL } \\
\hline$<130$ & 264 & $39.0(0.7)$ & $41.3(0.8)$ & $0.07(0.01)$ & 0.025 & 88 & $40.6(1.1)$ & $44.9(1.2)$ & $0.12(0.02)$ & 0.008 & $0.160^{\mathrm{b}}$ \\
\hline$\geq 130$ & 176 & $41.8(0.9)$ & $44.6(1.0)$ & $0.08(0.02)$ & 0.031 & 33 & $44.7(2.7)$ & 48.9 (3.5) & $0.09(0.03)$ & 0.344 & $0.802^{\mathrm{b}}$ \\
\hline$P$ value ${ }^{c}$ & & 0.011 & 0.006 & 0.474 & & & 0.161 & 0.291 & 0.489 & & $0.472^{\mathrm{d}}$ \\
\hline \multicolumn{12}{|l|}{$\mathrm{TG} \mathrm{mg} / \mathrm{dL}$} \\
\hline$<150$ & 192 & $44.0(1.1)$ & $46.5(1.1)$ & $0.08(0.02)$ & 0.102 & 60 & $46.6(1.6)$ & $50.4(2.2)$ & $0.08(0.02)$ & 0.166 & $0.899^{\mathrm{b}}$ \\
\hline$\geq 150$ & 483 & $38.0(0.4)$ & $40.9(0.5)$ & $0.08(0.01)$ & $<0.001$ & 93 & $37.6(1.0)$ & 41.8 (1.1) & $0.12(0.02)$ & 0.005 & $0.060^{\mathrm{b}}$ \\
\hline$P$ value ${ }^{c}$ & & $<0.001$ & $<0.001$ & 0.943 & & & $<0.001$ & 0.001 & 0.111 & & $0.362^{\mathrm{d}}$ \\
\hline \multicolumn{12}{|l|}{$\mathrm{TC} \mathrm{mg} / \mathrm{dL}$} \\
\hline$<200$ & 301 & $36.8(0.6)$ & $39.5(0.6)$ & $0.09(0.01)$ & 0.001 & 96 & $39.6(1.0)$ & $43.7(1.2)$ & $0.11(0.02)$ & 0.007 & $0.362^{b}$ \\
\hline$\geq 200$ & 375 & $42.2(0.6)$ & $44.9(0.7)$ & $0.08(0.01)$ & 0.004 & 57 & 43.7 (1.9) & $47.6(2.4)$ & $0.10(0.03)$ & 0.200 & $0.530^{\mathrm{b}}$ \\
\hline$P$ value ${ }^{c}$ & & $<0.001$ & $<0.001$ & 0.259 & & & 0.052 & 0.144 & 0.549 & & $0.195^{\mathrm{d}}$ \\
\hline
\end{tabular}

Definition of lipids: HDL-C: high-density lipoprotein cholesterol; LDL-C: low-density lipoprotein cholesterol; TG: triglycerides; TC: total cholesterol.

${ }^{a} P$ value tested the null hypothesis that there is no difference in the baseline and follow-up HDL-C levels using the Student's $t$-test.

${ }^{\mathrm{b}} P$ value tested the null hypotheses that there is no difference in the mean HDL-C effect across niacin ER dose groups using the Student's $t$-test.

${ }^{c} P$ value tested the null hypothesis that there is no difference in the mean HDL-C effect across each risk factor/lipid level category using the Student's $t$-test.

${ }^{\mathrm{d}} P$ value tested the null hypothesis that there is no difference in the mean HDL-C effect across each risk factor/lipid level category using the $F$-statistic.

$P=0.001)$ and among individuals with total cholesterol levels $\geq 200 \mathrm{mg} / \mathrm{dL}$ (mean HDL-C effect $=0.08, P=0.004$ ) in the $500 \mathrm{mg}$ niacin ER dose group, but only among individuals with total cholesterol levels $<200 \mathrm{mg} / \mathrm{dL}$ in the $1000 \mathrm{mg}$ niacin ER dose group (mean HDL-C effect $=0.011, P=0.007$ ).

Table 3 summarizes the results of the univariate and multivariate logistic regression models that evaluated the predictors of high response to the $500 \mathrm{mg}$ dose of niacin ER. The only statistically significant predictors of response to niacin ER in the univariate models were triglyceride levels $>150 \mathrm{mg} / \mathrm{dL}$ and low HDL-C levels $(<40 \mathrm{mg} / \mathrm{dL}$ for males and $<50 \mathrm{mg} / \mathrm{dL}$ for females). Triglyceride levels did not maintain statistical significance in the multivariate model. However, both males and females with low HDL-C levels exhibited a strong response to treatment in the multivariate model (males, HDL-C <40 mg/dL: OR = 5.18, 95\% CI: $2.36-$ 11.39; females, HDL-C <50 mg/dL: OR $=5.40$, 95\% CI: 1.84-15.79). Similar, but attenuated, trends were observed in the multivariate sensitivity analysis model (males, HDL-C $<40 \mathrm{mg} / \mathrm{dL}: \mathrm{OR}=4.90,95 \% \mathrm{CI}: 1.56-15.46$; females, HDL-C $<50 \mathrm{mg} / \mathrm{dL}: \mathrm{OR}=2.34,95 \% \mathrm{CI}: 0.61-9.04$ ) (data not shown).

\section{Discussion}

Approximately one-quarter of the adult population in the USA has low HDL-C [8]. The causes of low HDL-C levels are largely unknown; however, there is evidence to suggest that being overweight or obese, having limited physical activity, smoking, increased intake of carbohydrates, Type II diabetes, 
TABle 3: Predictors of response to niacin ER (500 mg dose).

\begin{tabular}{|c|c|c|c|c|}
\hline & $\begin{array}{l}\text { High responder } \\
\qquad \begin{array}{c}1 \\
n=202) \\
n(\%)\end{array}\end{array}$ & $\begin{array}{c}\text { Low responder }{ }^{2} \\
(n=305) \\
n(\%)\end{array}$ & Crude OR $(95 \% \mathrm{CI})^{3}$ & Adjusted OR $(95 \% \mathrm{CI})^{3,4}$ \\
\hline Age (years, continuous) & - & - & $0.10(0.97-1.02)$ & $1.0(0.97-1.03)$ \\
\hline \multicolumn{5}{|l|}{ Gender } \\
\hline Female & $39(32.5 \%)$ & $53(29.1 \%)$ & 1.0 & 1.0 \\
\hline Male & $81(67.5 \%)$ & $129(70.9 \%)$ & $0.85(0.52-1.40)$ & $0.79(0.25-2.51)$ \\
\hline \multicolumn{5}{|c|}{ HDL-C mg/dL (males only) } \\
\hline$\geq 40$ & $49(40.8 \%)$ & $111(61.0 \%)$ & 1.0 & 1.0 \\
\hline 40 & $71(59.2 \%)$ & $71(39.0 \%)$ & $2.27(1.42-3.63)$ & $5.18(2.36-11.39)$ \\
\hline \multicolumn{5}{|c|}{ HDL-C mg/dL (females only) } \\
\hline$\geq 50$ & $87(72.5 \%)$ & $155(85.2 \%)$ & 1.0 & 1.0 \\
\hline$<50$ & $33(27.5 \%)$ & $27(14.8 \%)$ & $2.18(1.23-3.86)$ & $5.40(1.84-15.79)$ \\
\hline \multicolumn{5}{|l|}{ LDL-C mg/dL } \\
\hline$<130$ & $69(57.5 \%)$ & $103(56.6 \%)$ & 1.0 & 1.0 \\
\hline$\geq 130$ & $51(42.5 \%)$ & $79(43.4 \%)$ & $0.96(0.61-1.54)$ & $1.23(0.54-2.77)$ \\
\hline \multicolumn{5}{|l|}{$\mathrm{TG} \mathrm{mg} / \mathrm{dL}$} \\
\hline$<150$ & $32(26.7 \%)$ & $71(39.0 \%)$ & 1.0 & 1.0 \\
\hline$\geq 150$ & $88(73.3 \%)$ & $111(61.0 \%)$ & $1.76(1.06-2.91)$ & $1.38(0.76-2.50)$ \\
\hline \multicolumn{5}{|l|}{$\mathrm{TC} \mathrm{mg} / \mathrm{dL}$} \\
\hline$<200$ & $62(51.7 \%)$ & $85(46.7 \%)$ & 1.0 & 1.0 \\
\hline$\geq 200$ & $58(48.3 \%)$ & $97(53.3 \%)$ & $0.82(0.52-1.30)$ & $1.0(0.42-2.34)$ \\
\hline \multicolumn{5}{|l|}{ Diabetes } \\
\hline No & $82(68.3 \%)$ & $138(75.8 \%)$ & 1.0 & 1.0 \\
\hline Yes & $38(31.7 \%)$ & $44(24.2 \%)$ & $1.45(0.87-2.43)$ & $1.41(0.78-2.52)$ \\
\hline \multicolumn{5}{|l|}{ Stroke (within 6 months) } \\
\hline No & $114(95.0 \%)$ & $174(95.6 \%)$ & 1.0 & 1.0 \\
\hline Yes & $6(5.0 \%)$ & $8(4.4 \%)$ & $1.15(0.39-3.39)$ & $0.69(0.19-2.49)$ \\
\hline \multicolumn{5}{|c|}{ Hypertension (within 6 months) } \\
\hline No & $108(90.0 \%)$ & $171(94.0 \%)$ & 1.0 & 1.0 \\
\hline Yes & $12(10.0 \%)$ & $11(6.0 \%)$ & $1.73(0.74-4.05)$ & $1.91(0.71-5.15)$ \\
\hline \multicolumn{5}{|c|}{ Ischemic heart disease (within 6 months) } \\
\hline No & 97 (80.8\%) & $147(80.8 \%)$ & 1.0 & 1.0 \\
\hline Yes & $23(19.2 \%)$ & 35 (19.2\%) & $1.0(0.56-1.79)$ & $0.91(0.44-1.87)$ \\
\hline \multicolumn{5}{|l|}{ Statin use during baseline } \\
\hline No & $50(41.7 \%)$ & $63(34.6 \%)$ & 1.0 & 1.0 \\
\hline Yes & $70(58.3 \%)$ & 119 (65.4\%) & $0.74(0.46-1.19)$ & $0.69(0.37-1.31)$ \\
\hline \multicolumn{5}{|c|}{ Fibric acid use during Baseline } \\
\hline No & $10(8.3 \%)$ & $22(12.1 \%)$ & 1.0 & 1.0 \\
\hline Yes & $110(91.7 \%)$ & $160(87.9 \%)$ & $1.51(0.69-3.32)$ & $1.60(0.69-3.75)$ \\
\hline
\end{tabular}

Definition of lipids: HDL-C: high-density lipoprotein cholesterol; LDL-C: low-density lipoprotein cholesterol; TG: triglycerides; TC: total cholesterol.

${ }^{1}$ High responder: HDL-C effect $>15 \%$.

${ }^{2}$ Low responder: HDL-C effect $<5 \%$.

${ }^{3}$ Outcome is high responder.

${ }^{4}$ Adjusted model includes all variables listed in the table. 
HDL effect

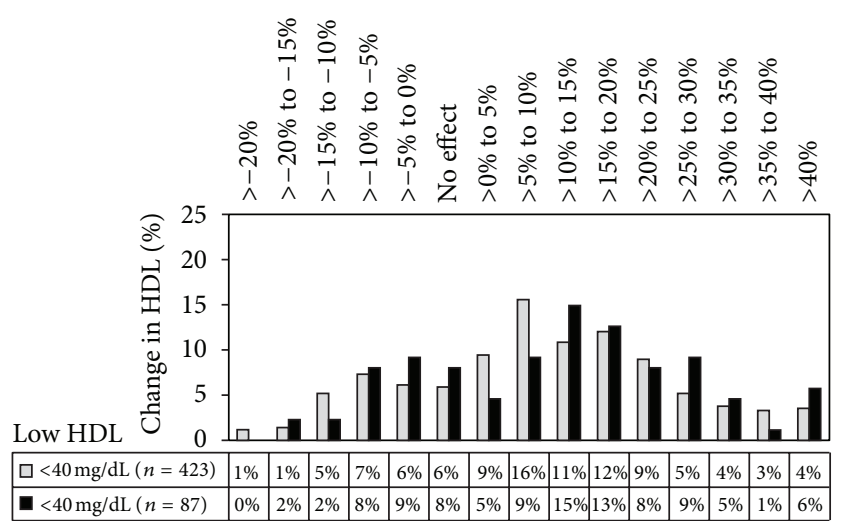

(a)

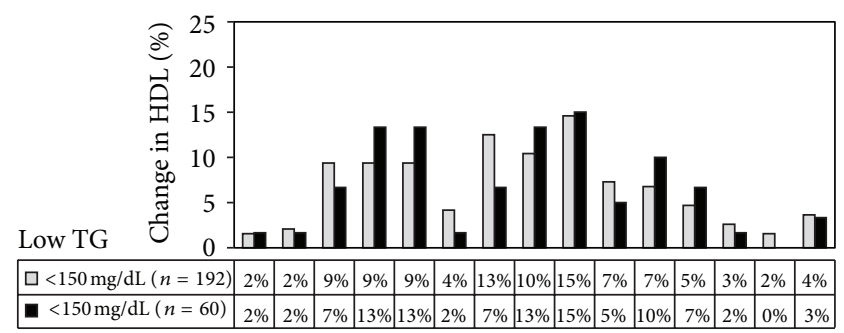

(c)

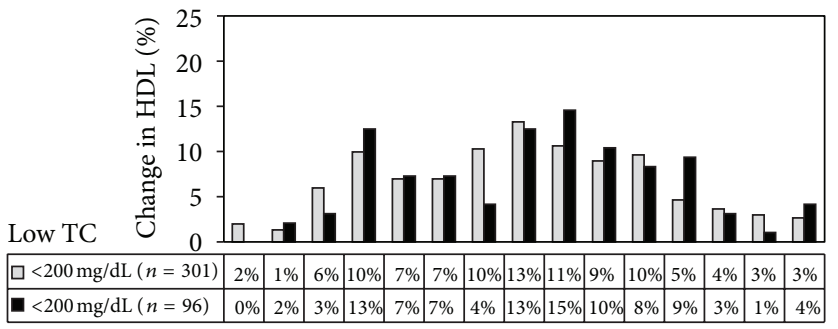

(e)

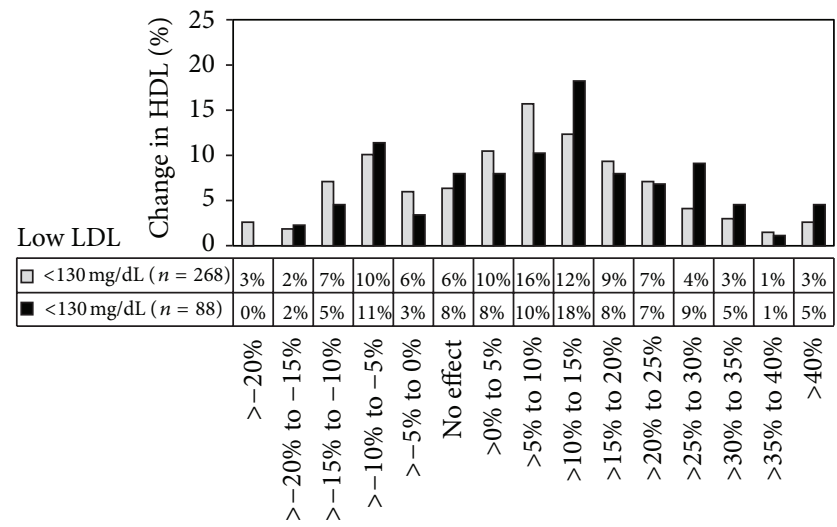

HDL effect

$\square$ Niacin $500 \mathrm{mg}$ dose group

- Niacin $1000 \mathrm{mg}$ dose group

(g)
HDL effect

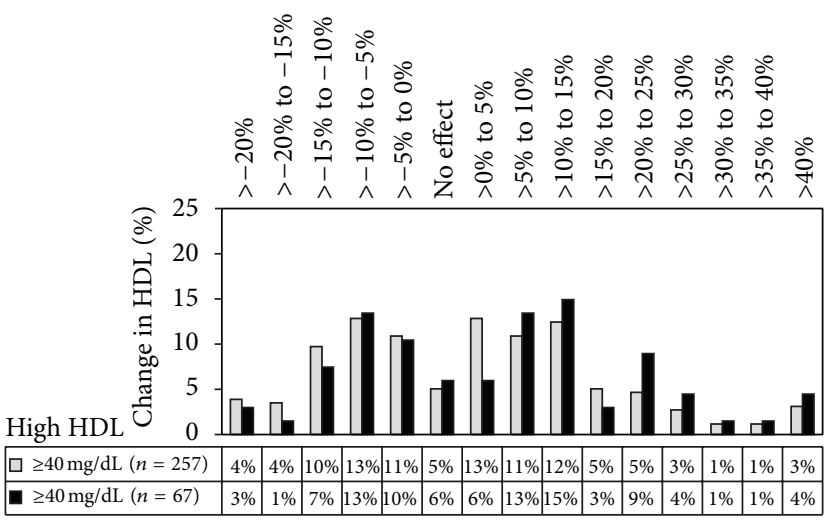

(b)

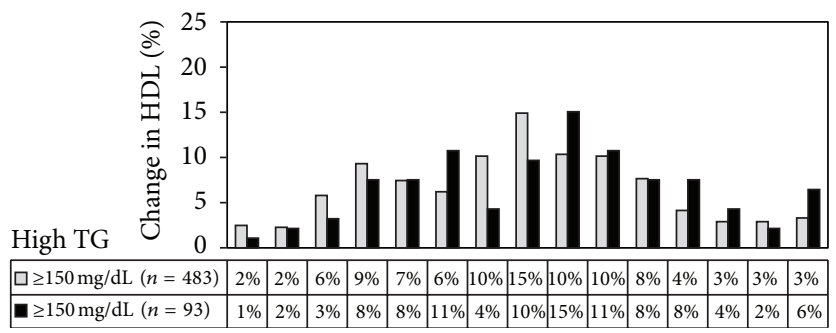

(d)

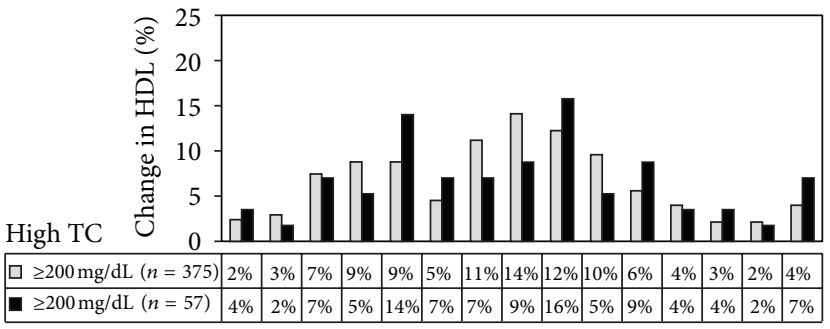

(f)

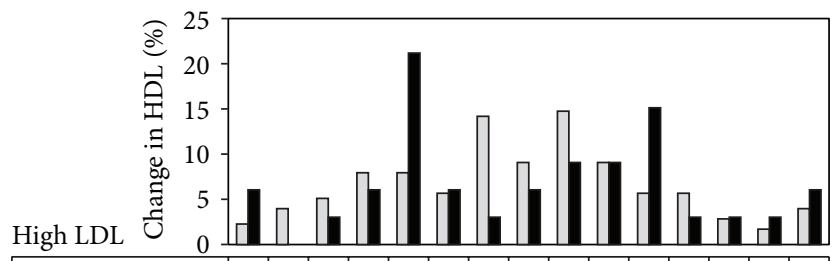

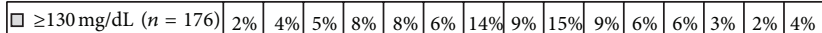
\begin{tabular}{|l|l|l|l|l|l|l|l|l|l|l|l|l|l|l|l|l|}
\hline - $\geq 130 \mathrm{mg} / \mathrm{dL}(n=33)$ & $6 \%$ & $0 \%$ & $3 \%$ & $6 \%$ & $21 \%$ & $6 \%$ & $3 \%$ & $6 \%$ & $9 \%$ & $9 \%$ & $15 \%$ & $3 \%$ & $3 \%$ & $3 \%$ & $6 \%$ \\
\hline
\end{tabular}

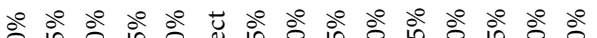

은

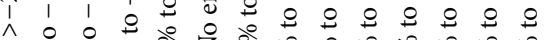

응

定 i⿱

$\stackrel{1}{1} \sqrt{1}$

HDL effect

$\square$ Niacin $500 \mathrm{mg}$ dose group

- Niacin $1000 \mathrm{mg}$ dose group

(h)

Figure 1: HDL effect by niacin ER dose and baseline lipid levels: (a) low HDL ( $<40 \mathrm{mg} / \mathrm{dL}$ ), (b) high HDL ( $>40 \mathrm{mg} / \mathrm{dL})$, (c) low TG $(<150 \mathrm{mg} / \mathrm{dL})$, (d) high TG $(>150 \mathrm{mg} / \mathrm{dL})$, (e) low TC (<200 mg/dL), (f) high TC ( $>200 \mathrm{mg} / \mathrm{dL})$, (g) low LDL (<130 mg/dL), and (h) high LDL ( $\geq 130 \mathrm{mg} / \mathrm{dL})$. 
genetic factors, and the use of some medications (i.e., betablockers, anabolic steroids, and progestational agents) may increase the likelihood of having low HDL-C [13]. A recent evaluation of data from the National Health and Nutrition Examination Survey (NHANES) identified several factors that were associated with HDL-C levels among both men and women. Specifically, being obese, having high apolipoprotein B levels $(>117 \mathrm{mg} / \mathrm{dL})$, and having high triglyceride levels $(\geq 200 \mathrm{mg} / \mathrm{dL}$ ) were shown to be associated with low HDL$C$ levels, while increased age (65 years or older) and having elevated total cholesterol ( $>240 \mathrm{mg} / \mathrm{dL}$ ) were shown to be associated with elevated HDL-C levels [4].

The clinical management of HDL-C is a somewhat vexing topic for several reasons. First, treatment with dyslipidemic pharmacotherapy, primarily niacin, has been shown to increase HDL-C levels by as much as $35 \%$ in some populations [6-9]. However, the manner in which these positive increases translate into reduced morbidity and mortality has been disappointing in recent studies. Results from the AIM-HIGH study showed a lack of efficacy in reducing cardiovascular-related events after 3.5 years, which led to the early termination of this study [4]. Second, niacin is known to cause cutaneous vasodilatation with flushing, which may include itching, tingling, warmth, and redness, which can lead to poor adherence or early discontinuation $[14,15]$. A cohort study of 2,369 individuals who were newly prescribed dyslipidemic agents reported a one-year probability of discontinuation of $46 \%$ for niacin versus $15 \%$ for lovastatin $[4,16]$.

Overall, our results showed that treatment with niacin ER at both the $500 \mathrm{mg}$ and $1000 \mathrm{mg}$ dose levels significantly increased HDL-C levels in both men and women. However, the average increase we observed was much lower than the $35 \%$ increase that has been observed in some populations. In our study, females had significantly higher levels of both baseline and follow-up HDL-C levels than males in both niacin ER dose groups. Furthermore, there was a statistically significant difference in the HDL-C effect observed among females across the niacin ER dose groups. This trend was not observed among males. When the association between age and effect of treatment was evaluated, the only statistically significant change in the mean HDL-C effect between baseline and follow-up HDL-C levels in both niacin ER dose groups was among individuals of 41 to 65 years of age. The mean HDL-C levels were statistically significantly higher in the $>66$-year age group compared with the younger age groups at both baseline and follow-up HDL-C measurements among participants in the $500 \mathrm{mg}$ niacin ER dose group but not in the $1000 \mathrm{mg}$ niacin ER dose group.

It is because of these paradoxical associations that we sought to identify factors that predict response to niacin ER, with the goal of providing useful information that may lead to the most efficient and successful prescription patterns for this treatment. Unfortunately, we were not able to conduct univariate and multivariate models for those treated with niacin ER at the $1000 \mathrm{mg}$ dose due to a smaller sample size. However, we did find that baseline HDL-C levels were the only factor that significantly predicted a high response to treatment with niacin ER at the $500 \mathrm{mg}$ dose level in the multivariate models. These results provide some insight into the factors that are associated with the effect of niacin treatment.

Limitations of our study may include the manner in which high response to niacin ER was classified. High response was defined as an HDL-C effect of $>15 \%$ and low response was defined as $<5 \%$ HDL-C effect. These thresholds were selected based on the distribution of response in our sample irrespective of characteristics and determined to be clinically meaningful responses. Modifications in these thresholds may have resulted in a slight change in our findings. However, we conducted a sensitivity analysis among people who were responding to taking their medications by including only those who had an LDL-C decrease of $15 \%$ or greater and our findings were consistent. Although monitoring prescription fills and biomarker response is not identical to consuming the medication, this method was the most accurate way of estimating actual medication use in a large population. In addition, clinical variables such as body mass index, exercise, diet, and smoking were not available in this healthcare claims database. We did not include other medications that may control HDL-C levels, such as statins, in our models. However, the majority of the patients included in this study were taking simvastatin at $20 \mathrm{mg}$ or $40 \mathrm{mg}$ doses. There is evidence to suggest that statins (as a drug class) only modestly increase HDL-C levels, with less than a $10 \%$ increase reported in two studies $[16,17]$. Finally, the quality control of laboratory measurements is assumed but cannot be verified. The laboratory values provided in the database are those that were provided by the laboratory vendor. As such, the specific laboratory methods or reference ranges that correspond with the lipid values are not available. Despite these limitations, the strengths of this study include the large sample size, which allowed us to evaluate a highly stratified matrix of response to treatment.

In summary, individuals with low HDL-C levels at baseline may have an increased risk of CHD-related events; however, the issues surrounding the pharmacological management of HDL-C are complex. We have shown that low baseline HDL-C levels are the strongest predictor of high response from the treatment of niacin ER at the $500 \mathrm{mg}$ dose level, among statin-treated individuals. We have also shown that both men and women had a significant increase in the mean HDL-C effect between baseline and followup, regardless of niacin ER dose. These findings can help clinicians and researchers better understand the extent to which response to niacin ER is variable and the importance of baseline HDL-C influencing response size while further data is generated to elucidate the value of HDL-C-raising therapy.

\section{Acknowledgments}

All the authors met the criteria for authorship set forth by the International Committee for Medical Journal Editors. J. B. Christian and E. J. Olson made substantial contributions to conception, study design, and interpretation of data. J. B. Christian and J. K. Allen were responsible for the acquisition of data and analytic plan. K. A. Lowe was responsible for 
preparing the figures and tables, drafting a significant portion of the paper, and providing editorial support. J. B. Christian, E. J. Olson, and J. K. Allen are employees of and hold equity interest in GlaxoSmithKline. None of the authors have a financial conflict associated with paper. Editorial support in the form of development of draft outline and first draft of paper, editorial suggestions to draft versions of this paper, assembling tables and figures, collating author comments, copyediting, fact checking, and referencing were provided by Exponent Health Sciences and was funded by GlaxoSmithKline.

\section{References}

[1] B. J. Ansell, K. E. Watson, A. M. Fogelman, M. Navab, and G. C. Fonarow, "High-density lipoprotein function: recent advances," Journal of the American College of Cardiology, vol. 46, no. 10, pp. 1792-1798, 2005.

[2] A. Morrison and J. E. Hokanson, "The independent relationship between triglycerides and coronary heart disease," Vascular Health and Risk Management, vol. 5, pp. 89-95, 2009.

[3] E. J. Schaefer, S. Lamon-Fava, J. M. Ordovas et al., "Factors associated with low and elevated plasma high density lipoprotein cholesterol and apolipoprotein A-I levels in the Framingham Offspring Study," Journal of Lipid Research, vol. 35, no. 5, pp. 871-882, 1994.

[4] W. E. Boden, J. L. Probstfield, T. Anderson et al., "Niacin in patients with low HDL cholesterol levels receiving intensive statin therapy," The New England Journal of Medicine, vol. 365, no. 24, pp. 2255-2267, 2011.

[5] "Prevalence of abnormal lipid levels among youths-United States, 1999-2006," MMWR. Morbidity and Mortality Weekly Report, vol. 59, no. 2, pp. 29-33, 2010.

[6] R. S. Birjmohun, J. J. P. Kastelein, D. Poldermans, E. S. G. Stroes, U. Hostalek, and G. Assmann, "Safety and tolerability of prolonged-release nicotinic acid in statin-treated patients," Current Medical Research and Opinion, vol. 23, no. 7, pp. 17071713, 2007.

[7] P. P. Toth, V. Zarotsky, J. M. Sullivan, and D. Laitinen, "Niacin and fibrate use among patients with high triglycerides and low high-density lipoprotein cholesterol," Current Medical Research and Opinion, vol. 25, no. 6, pp. 1355-1363, 2009.

[8] J. B. Christian, N. E. Bourgeois, and K. A. Lowe, "Prevalence, clinical characteristics and treatment patterns of low highdensity lipoprotein cholesterol in the US population: National Health and Nutrition Examination Survey 2005-2008," Journal of Cardiovascular Medicine, vol. 12, no. 10, pp. 714-722, 2011.

[9] P. P. Toth, V. Zarotsky, J. M. Sullivan, and D. Laitinen, "Dyslipidemia treatment of patients with diabetes mellitus in a US managed care plan: a retrospective database analysis," Cardiovascular Diabetology, vol. 8, article 26, 2009.

[10] B. Alemayehu, E. Buysman, D. Parry, L. Becker, and F. Nathan, "Economic burden and healthcare utilization associated with castration-resistant prostate cancer in a commercial and Medicare Advantage US patient population," Journal of Medical Economics, vol. 13, no. 2, pp. 351-361, 2010.

[11] J. B. Christian, B. Arondekar, E. K. Buysman et al., "Clinical and economic benefits observed when follow-up triglyceride levels are less than $500 \mathrm{mg} / \mathrm{dL}$ in patients with severe hypertriglyceridemia," Journal of Clinical Lipidology, vol. 6, no. 5, pp. 450461, 2012.
[12] S. A. Williams, E. K. Buysman, E. M. Hulbert et al., "Hemoglobin Alc outcomes and health care resource use in type 2 diabetes mellitus patients treated with combination oral antidiabetic drugs through step therapy and loose-dose and fixed-dose combinations," Managed Care, vol. 21, pp. 40-48, 2012.

[13] "Third Report of the National Cholesterol Education Program (NCEP) Expert Panel on Detection, Evaluation, and Treatment of High Blood Cholesterol in Adults (Adult Treatment Panel III) final report," Circulation, vol. 106, no. 25, pp. 3143-3421, 2002.

[14] T. A. Jacobson, "A "hot" topic in dyslipidemia management"how to beat a flush": optimizing Niacin tolerability to promote long-term treatment adherence and coronary disease prevention," Mayo Clinic Proceedings, vol. 85, no. 4, pp. 365-379, 2010.

[15] S. Kamal-Bahl, D. J. Watson, and B. M. Ambegaonkar, "Patients' experiences of niacin-induced flushing in clinical practice: a structured telephone interview," Clinical Therapeutics, vol. 31, no. 1, pp. 130-140, 2009.

[16] C. L. Shear, F. A. Franklin, S. Stinnett et al., "Expanded Clinical Evaluation of Lovastatin (EXCEL) Study results: effect of patient characteristics on lovastatin-induced changes in plasma concentrations of lipids and lipoproteins," Circulation, vol. 85, no. 4, pp. 1293-1303, 1992.

[17] A. S. Wierzbicki and D. P. Mikhailidis, "Dose-response effects of atorvastatin and simvastatin on high-density lipoprotein cholesterol in hypercholesterolaemic patients: a review of five comparative studies," International Journal of Cardiology, vol. 84, no. 1, pp. 53-57, 2002. 


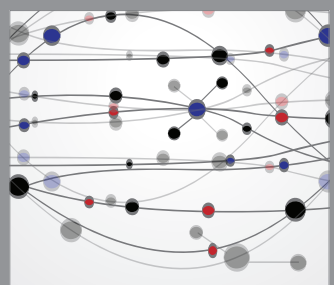

The Scientific World Journal
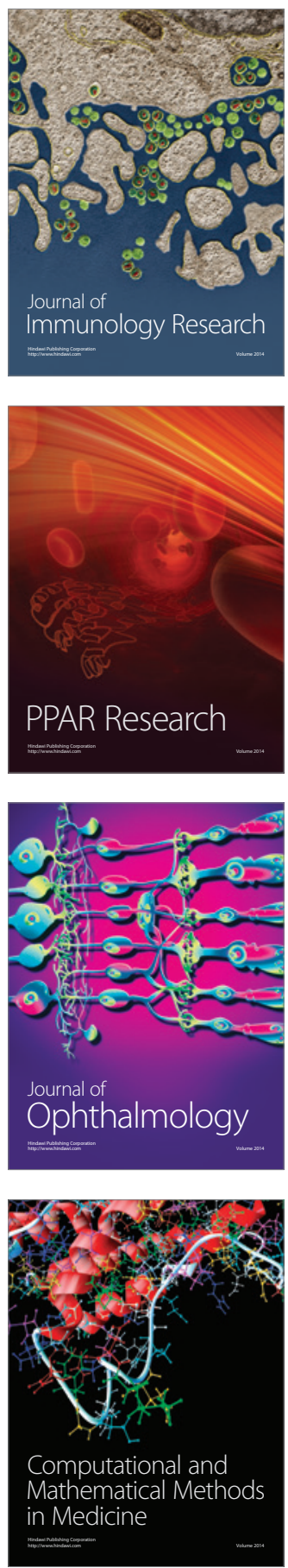

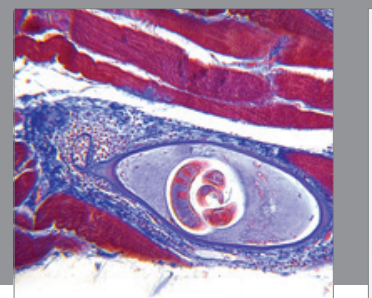

Gastroenterology

Research and Practice
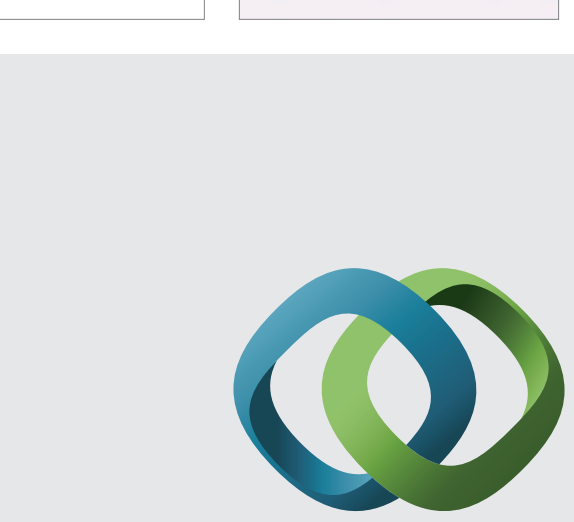

\section{Hindawi}

Submit your manuscripts at

http://www.hindawi.com
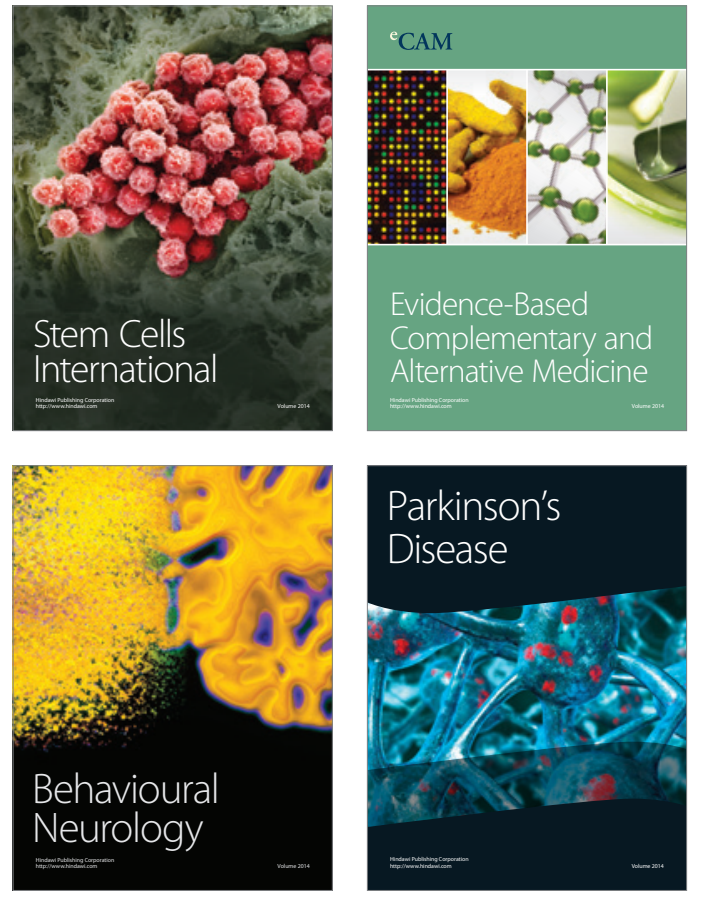
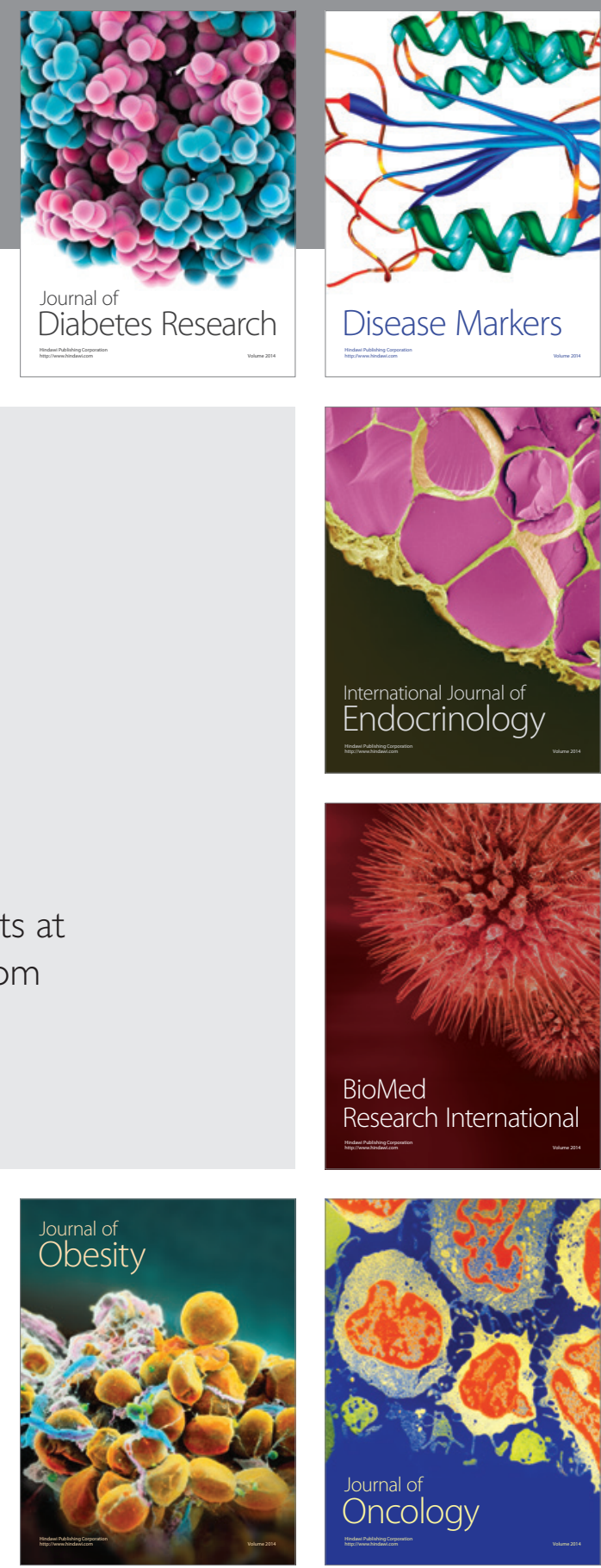

Disease Markers
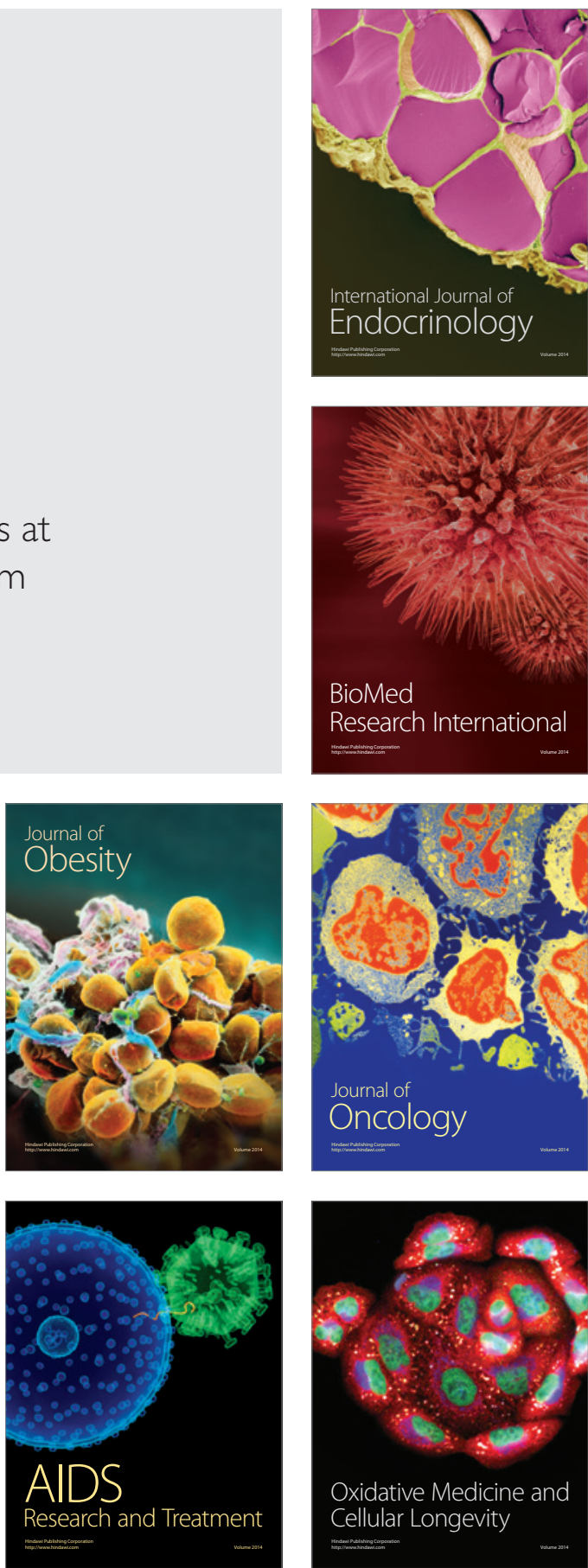